\section{'Can I stop even one of these pills?' The development of a tool to make deprescribing easier}

\author{
Alan Cassels
}

While everyone might recognise the problems inherent in polypharmacy in the elderly, especially due to its many potential harms, there seems to be little consensus on how to reduce the medication burden on our older patients, and few tools to help clinicians initiate deprescribing.

The medical system often supports interventions and shared decision-making in the introduction of evidence-based treatments, yet the literature suggests that without a framework to support polypharmacy risk reduction activities, clinicians do not feel confident about initiating 'deprescribing.'

The prospect of deprescribing is fraught with considerable emotional and psychological stress for both practitioners and their patients. Previous studies ${ }^{1-3}$ have shown that many patients believe they are taking too many medications, yet despite the belief, there are barriers to asking their clinician to reduce their number of prescriptions. Patients fear their clinician's response, ${ }^{3}$ fear relapsing, ${ }^{4}$ fear being denied the ability to resume medicating ${ }^{5}$ and fear abandonment by their healthcare provider. ${ }^{4}$ Conversely, clinicians admit that deprescribing comes with anxiety, including uncertainty over why drugs were prescribed in the first place, ${ }^{6}$ fear that deprescribing will add to workload pressures $^{5}$ and reluctance to engage patients in discussing quality-of-life/life expectancy issues. ${ }^{4}$ Clinicians have some unease about their ability to manage patients' discontinuation of medications and fear conflict with other healthcare providers. $^{7}$

Despite these barriers, one irrefutable aspect in any discussion of deprescribing is its potential for overwhelming benefit. It can be, in the words of a group of New Zealand researchers, an 'antidote' to polypharmacy's many harms and write that deprescribing is "associated with numerous health benefits including improvement in cognition, a reduction in falls, a decrease in fractures, better medication

Correspondence to Dr Alan Cassels, University of Victoria, 423 Stannard Avenue, Victoria, British Columbia, Canada V8S 3M6; cassels@uvic.ca adherence, and improvement in quality of life". 8

Interestingly, and perhaps more problematically, no comprehensive guidelines to date have been designed specifically to guide deprescribing. Deprescribing takes place in a relatively evidence-free zone. Why? There are no randomised controlled trials of adding or subtracting medications among patients taking multiple medications. Even if meta-analyses of trials prove drugs to be effective among younger, healthier patients, this may not translate into accurate predictions in how those drugs could benefit elderly polypharmacy patients. On the other hand, there is much clinical experience and several randomised trials showing improvements in functioning of polypharmacy patients after reducing the number of drugs they are taking.

While there are a number of resources that help clinicians identify specific medications that may have the potential to be inappropriate (BEERS, Drug Burden Index, Screening Tool of Older Persons' potentially inappropriate Prescriptions), there are very few resources available that provide an overall synopsis of the potential value of specific medications benefit/ harm or provide information as to how to stop or reduce the dose of a specific medication. When we asked clinicians in Continuing Medical Education sessions on polypharmacy whether they have a systematic approach to deprescribing, most admitted they did not have one. Therefore, we hypothesised that it might be feasible and useful to produce an interactive web-based, consensus-informed process to guide them in a systematic way.

Our team in Victoria and Vancouver, British Columbia, won a 2-year grant from the Canadian Institutes for Health Research, which was funded specifically to increase the uptake/application of knowledge by supporting partnerships between researchers and knowledge-users to bridge a knowledge to action gap. In this case, the knowledge-users were primary care clinicians and so we focused on developing a tool that could make deprescribing more deliberate, logical and easier to initiate.

The development of the MedStopper/ database tool began when James
McCormack, a professor in the Faculty of Pharmaceutical Sciences at the University of British Columbia, was faced with a question by a local general practitioner (GP) whose 90-year-old very frail grandmother, at that time on 23 medications and vitamin supplements, asked: "Can I stop even one of these pills?" The GP did not know how to initiate the needed deprescribing exercise, so asked James' opinion.

Solving this puzzle became the basis of MedStopper.

James proposed that the basis of MedStopper should be a list of a patient's drug-indication pairs, roughly ranked from potentially most stoppable to potentially least stoppable, with concise displays of the rationale (and evidence if available) for their ranking: a combination of indirect evidence from trials among healthier patients and clinical judgement of experienced deprescribers.

We created a draft MedStopper webpage to demonstrate the functions that we envisioned. The goal was to allow busy users, with a minimum of keystrokes, to select a patient's medications and their indications, and quickly see a visual ranking of the medication-indication pairs based on effectiveness and safety: short-term impacts on symptoms, evidence of longterm improvements in outcomes and potential harms.

The webpage interfaced with a spreadsheet of approximately 400 medications (brand/generic), placed into roughly 80 medication categories (eg, statins, selective serotonin reuptake inhibitors, ACE inhibitors). As specific medication categories can be used for a number of indications (blood pressure, heart failure, depression, chronic pain, etc), these 80 medication categories were expanded into 125 categories/indications that should include the majority of medications used in family practice.

In order to get the expertise and 'evidence' to populate this spreadsheet, we invited a group of experts in gerontology, polypharmacy, pharmacology, pharmacy, patient advocacy and family medicine to a two-day meeting in January 2015 in Vancouver and asked them to help us build a demonstration MedStopper webpage.

Over those two days, we went through each category/indication, asking our panel members to provide their opinion as to the value of each of the 125 categories/ indications based on three criteriasymptom control, long-term benefit and potential for harm. Each one of these criteria was ranked on a scale of 1-3. 
Most of the time there was strong agreement on the scoring of each question. When there was disagreement, the panel heard the arguments from both sides of the disagreement and worked towards a consensus opinion. With the scoring, we applied an algorithm to calculate a medication-specific score and linked to the indication for which the patient is taking the drug. The algorithm generated either a happy face, a neutral face or a sad face. In addition, these experts were asked to provide feedback as to how they would stop medications in these categories. Specifically they were asked for the tapering process and monitoring plan they would follow or if they would just stop the medication in the specific category/ indication. Those suggestions on stopping and tapering were recorded as part of our database. We used this database and medication scoring system to develop the website further.

As we translated the experts' judgements into values in the spreadsheet and represented the values in the webpage interface (http://www.medstopper.com), we confronted a series of design decisions that each required clarification of clinical and pharmacological issues. We were definitive that the ranking of the medications from 'most likely to stop' (coloured red and at the top) down to 'least likely to stop' (coloured orange or yellow and closer to the bottom) was to guide sequencing of thoughts, not sequencing of actions. The visual display was to give the clinicians something to look at and consider, not something that told them what to do.

We pledged that MedStopper should aim to improve, not to perfect, clinicians' thinking and decided along the way that "perfection must not be the enemy of good". For example, after one enters the medication name, to proceed one has to enter the indication. Often, however, the conditions that caused the starting medications are often not known. We added 'unknown' as an option knowing that this is a less than perfect solution, yet one that leaves room for the clinician to draw on knowledge of the patient and clinical instincts.

Our team was in unanimous agreement that at the start of any deprescribing exercise the clinician should seriously consider discontinuing any medication that is likely causing unnecessary symptoms and/or otherwise degrades the quality of life.

After we developed the database and the working algorithms in MedStopper, we took the tool and carried out a series of time-and-motion studies with clinicians in a long-term care facility. Student observers measured the length of time it took to input drugs and draw conclusions from the MedStopper results and concluded that it would serve as a time-saving tool. The most salient point we heard from the participants was that the tool would "be useful for discussions with families" on cutting back medications under the belief that these long-term elderly residents are often surrounded by very involved family members who may be reluctant to see their elderly parent taken off drugs.

We then conducted interviews with 18 physicians, polypharmacy leaders and those already skilled in doing med-reviews in long-term care. Generally, we found few objections to it: these interviewees told us the tool was easy to use, intuitive and generally helpful. The faces made sense though there were some questions on some drug areas (ie, statins). We gathered suggestions on improvements and incorporated them into a new version of the tool. Some requested additional indications, an automatic link to a cardiovascular disease risk calculator and a numbers needed to treat/numbers needed to harm generator all which we added. Most of the interviewees found the tapering advice useful yet some found the tapering recommendations too. Some suggestions that we should include on the next iteration more information on drug interactions, costs and more detail on side effects. Generally, the interviewees told us that prioritising drugs to cut was quite welcomed, and those physicians interviewed appreciated the feature that gave an 'at a glance' listing of the drugs by class.

Once we had completed the beta website, we launched it to the world. We conducted a social media blitz that featured a YouTube video we created, which showed the Beatles singing the Medstopper song (with lyrics to Day Tripper changed). We presented MedStopper at conferences such as the Preventing Overdiagnosis conference at the National Institutes of Health in Washington DC in September 2015. We have since been contacted by research projects in Canada and around the world, interested in partnering with MedStopper and creating a version that could be adaptable to electronic health records.

We realised the importance of validating the tool in a more rigorous trial and were lucky to learn that, together with a consortium of researchers in Canada lead by a group at McGill University, we have been awarded a $\$ 1.6$ million federal research grant to study and validate the MedStopper tool. That research will begin in the fall of 2016 . We hope that this research will prove the viability of our deprescribing tool and address what has been a shortcoming in proper rational prescribing.

As for the 90-year-old grandmother, she was able to reduce her medication load down to five medications, felt a lot better and appreciated the reprieve from taking all her medications. She lived another 6 months, mostly staying out of the hospital.

\section{EAHP Statement 6: Education and} Research

Twitter Follow Alan Cassels at @AKECassels

Contributors AC drafted the initial commentary and James McCormack made suggestions for improvement and helped revise the manuscript.

Competing interests None declared.

Provenance and peer review Not commissioned; externally peer reviewed.

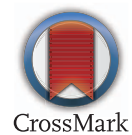

To cite Cassels A. Eur J Hosp Pharm 2017;24:3-4.

Eur J Hosp Pharm 2017;24:3-4.

doi:10.1136/ejhpharm-2016-001011

\section{REFERENCES}

1 Reeve $\mathrm{E}$, To $\mathrm{E}$, Hendrix I, et al. Patient barriers to and enablers of deprescribing: a systematic review. Drugs Aging 2013;30:793-807.

2 Reeve E, Shakib S, Hendrix I, et al. Review of deprescribing processes and development of an evidence-based, patient-centred deprescribing process Brit J Clin Phamacol 2014;78:738-47.

3 Linsky A, Simon SR, Bokhour B. Patient perceptions of proactive medication discontinuation. Patient Educ Couns 2015;98:220-5.

4 Scott IA, Anderson K, Freeman CR, et al. First do no harm: a real need to deprescribe in older patients. Med J Aust 2014;201:390-2.

5 Reeve E, Bell J, Hilmer S. Barriers to optimising prescribing and deprescribing in older adults with dementia: A narrative review. Curr Clin Pharmacol 2015;10:168-77.

6 Plakiotis C, Bell JS, Jeon YH, et al. Deprescribing psychotropic medications in aged care facilities: the potential role of family members. Advan Experim Med Biol 2015;821:29-43.

7 Anderson K, Stowasser D, Freeman C, et al. Prescriber barriers and enablers to minimizing potentially inappropriate medications in adults: a systematic review and thematic synthesis. BMJ Open 2014;4:e006544.

8 Ailabouni NJ, Nishtala PS, Mangin D, et al. Challenges and enablers of deprescribing: a general practitioner perspective. PLOS ONE 2016;11: e0151066. 